\title{
THE EVOLUTION OF CLOSE BINARIES AND THE Am STARS
}

\author{
Peter S. ConTI \\ (Lick Observatory, University of California, Santa Cruz, Calif., U.S.A.)
}

In this paper, I shall be concerned with three related questions: (a) what is the evolutionary fate of close binary systems, (b) is this concept consistent with the number of evolved close binary systems, and (c) are these evolved systems the Am stars?

For the first question, it should be kept in mind that as stars evolve to the giant region they greatly expand. The interesting point then arises, what is the value of the critical period, $P_{\mathrm{c}}$, for a given mass (and mass ratio), such that the primary in a close binary system with any period, $P \leqslant P_{\mathrm{c}}$, will reach its Roche limit during its post-mainsequence evolution? This can be answered numerically by using Kepler's law and writing it in the form:

$$
R_{0}=a r=4 \cdot 2 r\left(m_{1}+m_{2}\right)^{1 / 3} P^{2 / 3} .
$$

In Equation (1), the masses are in solar units, the period is in days, the mean separation, $a$, and Roche radius, $R_{0}$, are in solar radii, and $r$ is the Roche function tabulated by, say, Plavec and Kratochvil (1964). The Roche function takes on the values $0.37 \leqslant r \leqslant 0.60$ for the primary star for mass ratios $1 \cdot 0 \leqslant m_{2} / m_{1} \leqslant 0 \cdot 1$, respectively.

To simplify the discussion I adopt the value of 0.5 for $r$ (corresponding to a mass, it tio of 0.27 ), which is sort of a mean value for this quantity. Then, for a given mass ra is simple to find the period for any radius $R_{0}$. Since radii or their corresponding periods are related to the temperature and luminosity, they can be placed on an HR diagram. For this I used the temperature, $B-V$ and bolometric corrections of Johnson (1966). The result is Figure 1. Also shown are the evolutionary tracks of Iben (1966, 1967) transformed in a like manner. It is apparent that binaries of quite appreciable periods will reach their Roche limits during their evolution. The horizontal part of each track corresponds to the time when hydrogen burning in the core has ceased and a very rapid evolution is proceeding. Kippenhahn and Weigert (1967) distinguished three possible cases for close binary evolution. For case $a$, the primary reaches its lobe before the end of core hydrogen burning; for case $b$, the primary reaches the lobe after core hydrogen burning but before core helium burning; and for case $c$, the primary reaches its lobe after core helium burning.

As can be seen from Figure 1, only binaries with periods less than about 1 day will

Perek (ed.), Highlights of Astronomy, 437-442. (c) I.A.U. 


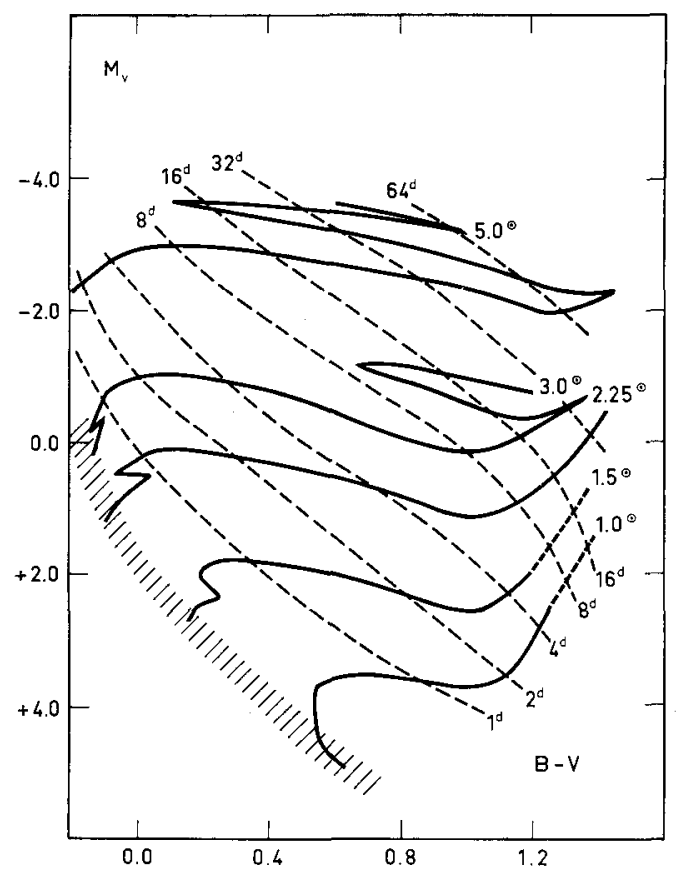

FIG. 1.

be in case $a$. Some binaries will reach case $c$, but a large number of known binaries with periods between 1 day and, say, 10-50 days will be in case $b$. Kippenhahn et al. (1967) have followed the detailed evolution of a system in case $b$. They find that if the primary is less massive than 2.80 , it transfers material to the secondary and goes directly to the white-dwarf state. We see this represents masses for the primary corresponding to $\mathrm{A}$ and $\mathrm{F}$ stars and periods up to at least 10 days. We will consider these systems only in what follows.

It is necessary to choose a sample of stars that have been reasonably well studied for duplicity. Suppose one picks the stars in the Bright Star Catalogue (BSC). There are 97 known spectroscopic binaries (SB) in this catalogue with periods less than 10 days, spectral type A or F. Unfortunately, the BSC stars have not been uniformly well studied for duplicity. The only complete study of a sample of stars for duplicity has been that of Abt $(1961,1965)$. Among a sample of late A-early F stars, he finds $5 \cdot 5 \%$ are short period binaries $(P<10$ days). If one assumes that this percentage would also apply to the early $A$ and late $F$ stars, then the total number of $A$ and $F$ short-period $\mathrm{SB}$ in the BSC would be 195.

Eclipsing semi-detached (SD) systems have been supposed to be the evolved close binaries (Crawford, 1955). From Kippenhahn et al. (1967) it is possible to estimate the time-scale for the semi-detached mode for the A and F short-period SBs. For 
their A star with a mass of $2 \odot$, the time on the main sequence (MS) is $5 \times 10^{8}$ years. This is to be compared with the (total) time as a SD system of $8.5 \times 10^{7}$ years. In other words, about $1 / 6$ of the MS lifetime is spent in the SD mode. Then, the number of SD systems of types $A$ and $F$ in the BSC should be $1 / 6$ the number of SBs. By actual count there are four identified SD systems, all eclipsing. This number may be underestimated by a small factor since there are several more 'Algol' types in the BSC not completely studied, and a few other EBs may have been undetected as yet. Perhaps there are five eclipsing SD systems. But this number is an underestimate of the total since eclipsing systems are preferentially viewed edge on. A factor five (Conti, 1967) more semi-detached systems are viewed non edge on. There are thus about 30 semidetached $\mathrm{A}$ and $\mathrm{F}$ systems in the BSC.

This expected total number of SD systems is within close to that predicted from the estimated number of short-period SB systems (195) and the time-scale arguments given by Kippenhahn et al. (1967) for case $b$ evolution. It is therefore legitimate to assert that the SD systems are the descendants of the short-period SB stars (at least for A and F spectral types). This must be so since the short-period SB stars will evolve, will fill their Roche lobes, will transfer material to the secondary, and/or will lose

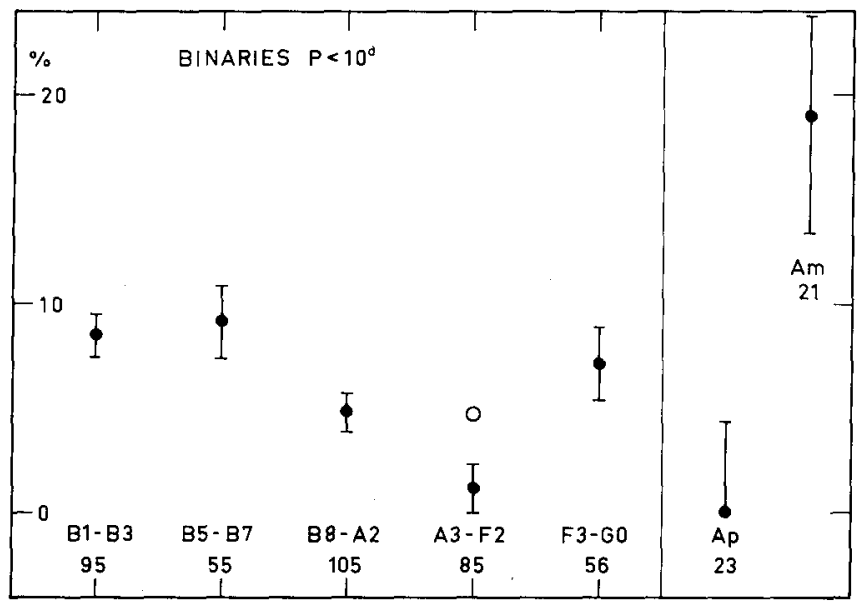

FIG. 2.

mass from the system. Then the observed small mass ratios of the SD systems, the overluminosity of the subgiant secondary, and the number of stars are all readily explained.

How do the Am stars fit in this evolutionary scheme? I will discuss only the shortperiod Am stars, which, according to Abt $(1961,1965)$, make up an appreciable frac- 
tion of these stars. If the Am stars were evolved systems, then we would expect them to be semi-detached, or at the next stage where they have white-dwarf companions (Conti, 1965; Van den Heuvel, 1967). A number of Am stars are double-lined. Out of the 27 identified Am short-period SB in the BSC, 7 have double lines. In none of these are the reported spectral types of the secondaries very different from the primaries. Therefore, at least $25 \%$ of the short-period Am stars are not semi-detached (nor do they have white-dwarf companions). $3 \mathrm{Am}$ stars (RR Lyn, WW Aur, and $\delta$ Cap) are eclipsing binaries, but all of these are detached systems. Additionally, none of the eclipsing SD systems known (see, e.g., Hack, 1963) are identified as Am systems. So there is no evidence that the Am stars are presently SD systems.

Is it possible that all Am stars (aside from the $25 \%$ with double lines) have whitedwarf companions? Let us suppose they all did have such companions and then examine the consequences. As such, there must be binaries on the main sequence for the first time which are their progenitors. Let us now look at the statistics of short-period binaries along the main sequence. It is most important to make a count of only those stars that have accurate and consistent spectral types. The only complete studies made, so far, have been those of Slettebak $(1954,1955)$ and Slettebak and Howard (1955), who gave MKK classifications and rotational velocities for nearly all stars brighter than $5^{\mathrm{m}} .0$ and North of $-20^{\circ}$. If we take these spectral types, and the fractional number of binaries known at that time (i.e. before Abt's work), we get Figure 2. I have used Slettebak's spectral type divisions (combining his last four groups into two) and listed the Am (and Ap) stars separately. The numbers below the spectral classes are the number of stars in that group; the vertical limits give the change in binary percentage for one more (or less) binary.

We see the result that from spectral classes $\mathrm{B} 1$ to $\mathrm{G} 0$ there is a sensibly constant percentage of binary stars, except in just one group, that of the A3-F2 stars. In fact, the one A3-F2 star in Figure 2, HD 118 216, is a non-eclipsing SD system (Conti, 1967). It would seem much more than a coincidence that it is just this group with practically no binaries that covers the spectral range of the Am stars. We see the well-known result that the percentage of Am stars is very high. It is only when the Am stars are added to the A3-F2 group that the fraction of short-period SB is the same in the A3-F2 group as in all the other spectral types (open circle in Figure 2). Unless one would care to assert that all short-period SB stars are evolved, or that there are no unevolved A3-F2 short-period SB stars, Figure 2 would force one to the conclusion that the short-period Am stars are not evolved. (Note this argument is not applicable for Ap stars.) If the short-period Am stars are not evolved, then the Am phenomena is not a product of post-main-sequence evolution.

One might ask, at this point, Isn't there an incompleteness in the numbers of known SB at all spectral types? This is true, but it should not completely change the arguments in the paragraph above since short-period binaries are those most easily detected. In any event, among almost the same group of A3-F2 stars, Abt $(1961,1965)$ has 
made a systematic study of duplicity. The results are well known: he finds the shortperiod binaries are all Am stars and he finds no normal A stars with periods less than 100 days! If we are to assert that the Am stars are not evolved, then the SD systems are their descendants. Since the SD systems show no Am anomalies, we conclude that the Am phenomena is confined to the outer layers of the star. This is consistent with arguments about rotation, depth of convective zone, and line strengths in Am stars advanced by Stromgren (1963) and others.

In a spirit of speculation, I would like to suggest one possible reason for the Am phenomenon, assuming it to be largely characterized by abundance anomalies. Going back to Figure 1, we visualize that close binaries pass through regions of contact interaction on the approach to the MS when on Hyashi convective tracks. It is known that the approach to the MS is a time of great surface activity for stars (e.g. T Tauri stars). There may be sufficient energy available for nuclear interactions in the region between the contracting stars. Although no detailed mechanisms are offered here, the contraction phase during the evolution of a star is at least as active, on the surface, as at any subsequent time.

This would suggest that Am stars will be found in the youngest clusters, e.g. NGC 2264. Although none have been found as yet, it should be kept in mind that few young clusters have been fully studied this far down on the MS. Also, one would suppose that a binary system is also a necessary condition for an Am star and only those clusters with short-period binaries will contain them. This has been suggested for the Pleiades (Abt et al., 1965), where there is only one marginal (classically defined) Am star, but where there are no short-period binaries among the late A stars, either.

\title{
References
}

\author{
Abt, H.A. (1961) Astrophys. J., Suppl., 6, 37. \\ Abt, H.A. (1965) Astrophys. J., Suppl., 11, 429. \\ Abt, H. A., Barnes, R.C., Biggs, E. S., Osmer, P.S. (1965) Astrophys. J., 142, 1604. \\ Conti, P.S. (1965) Astrophys. J., 142, 1594. \\ Conti, P.S. (1967) Astrophys. J., 149, 629. \\ Crawford, J. (1955) Astrophys. J., 121, 71. \\ Hack, M. (1963) Stellar Evolution, Academic Press, New York, p. 452. \\ Iben, I. (1966a) Astrophys. J., 142, 1447. \\ Iben, I. (1966b) Astrophys. J., 143, 283. \\ Iben, I. (1967a) Astrophys. J., 147, 624. \\ Iben, I. (1967b) Astrophys. J., 147, 650. \\ Johnson, H.L. (1966) A. Rev. Astr. Astrophys., 4, 193. \\ Kippenhahn, R., Weigert, A. (1967) Z. Astrophys., 65, 251. \\ Kippenhahn, R., Kohl, K., Weigert, A. (1967) Z. Astrophys., 66, 58. \\ Plavec, M., Kratochvil, P. (1964) Bull. Astr. Inst. Csl., 15, 165. \\ Slettebak, A. (1954) Astrophys. J., 119, 146. \\ Slettebak, A. (1955) Astrophys. J., 121, 653. \\ Slettebak, A., Howard, R. (1955) Astrophys. J., 121, 102. \\ Stromgren, B. (1963) Quart. J. R.A.S., 4, 8. \\ Van den Heuvel, E.P.J. (1967) Bull. astr. Inst. Netherl., 19, 11.
}




\section{DISCUSSION}

Van den Heuvel: You say that when the Am star becomes semi-detached, the Am character disappears. Do you agree that blue stragglers in galactic clusters descend from semi-detached systems? How can you then explain the fact that Pesch recently found that two blue stragglers in the old galactic cluster M 67 are Am stars? Then the Am character would only be absent during the s-d stage and reappear after the disappearance of the subgiant companion. Does not this seem strange?

Conti: I think the statement that there exist two Am stars in M 67 is not greeted by universal agreement among spectroscopists. 